\title{
Creation of Innovative Model of a Vertically Integrated Corporate Group with Regard to Optimality Properties
}

\author{
Lapshina M.L.* \\ Voronezh State University of Forestry and Technologies \\ named after G.F. Morozov \\ Voronezh, Russia \\ e-mail: marina_lapshina@mail.ru
}

Starikov A.V.

Voronezh State University of Forestry and Technologies named after G.F. Morozov

Voronezh, Russia

e-mail: star123@yandex.ru

\section{Gribanov A.A.}

Voronezh State University of Forestry and Technologies named after G.F. Morozov

Voronezh, Russia

e-mail:vgltaapp@mail.ru

\author{
Mescheryakova A.A. \\ Voronezh State University of Forestry and Technologies \\ named after G.F. Morozov \\ Voronezh, Russia \\ e-mail: aam_mtd_vglta@mail.ru
}

Pisareva S.V.

Voronezh State University of Forestry and Technologies named after G.F. Morozov

Voronezh, Russia

e-mail: pisareva_s@mail.ru

Budkova S.V.

Voronezh branch of State University of the Sea and River Fleet named after Admiral S.O. Makarova

Voronezh, Russia

e-mail:svb262@gmail.com

selection of innovative projects for their most effective future implementation. The results obtained can be interpreted as the ability of a corporate group to increase the efficiency of providing material resources for industrial research projects.

Keywords - innovative model, efficiency evaluation method, group, optimality.

\section{INTRODUCTION}

Works [1-2] confirm the possibility of using an optimal strategy for investing in innovative projects, based on the model of economic dynamics, which is based on the possibility of uneven changes in the technological process depending on the content of the innovation Fund. It is confirmed that in the case of a large horizon, the optimal strategy is close to the main-type problem. Works [3-4], based on an analysis of the development prospects of large financial and industrial associations specify their essential role as the basis for industrial development and support of innovative processes in the future economic perspective.

A significant fact that hinders the solution of problems of using achievements in science and technology in production processes is the use of commodity exchange in mutual settlements between enterprises. In [5] the analysis of such commodity exchange in the form of formed economic relationships is presented. It is proved that such aspects of business partnership in the economy as interest in partnership, interpersonal relations, and cooperation play a key role in the economic relations of corporate groups. The analysis of the 
model of behavioral features of a production enterprise that has certain difficulties with working capital confirmed that new incentives to join corporate groups arise in the conditions of non-payments [6].

\section{METHOD OF RESEARCH}

Each stage of the creation of the model considers the possibility of finding the optimal time intervals for financing by the Bank and the manufacturer, reducing the unit costs for a specific production, taking into account the shareholder relations that give the maximum criteria for economic efficiency. We will assume that in the analyzed model, the Bank that plays the role of the main investor occupies a priority position.

The optimal solution will be found when the equilibrium state is found in a differential game with non-antagonistic interests, as well as when the sequence of moves is set. The hermeyer principle of rational choice helps to achieve balance in the game [6] and guarantees the achievement of the required results for each participant. This approach was already implemented in the study of the dynamic model [7], but then the innovations used were not taken into account, which introduce certain nuances, which will be taken into account when building a dynamic model.

We have fixed the I-parameters corresponding to the level of material support for the implementation of innovative aspects, and we will find the optimal management intervals.

Stage I. The dynamics of the process

At the first stage we describe the dynamics of the process will be described using an ordinary differential equation of the form:

$$
x(t)=K_{1}(t)+V_{1}(t)
$$

and the corresponding boundary conditions

$$
x\left(t_{0}\right)=x_{0} ; t \in\left[t_{0}, T^{*}\right], x \in X, K_{1}(t) \in K, \quad K_{1}(t) \geq 0
$$

the Bank's management, $V_{1}(t) \geq 0$ - business management A.

Optimal strategies will be found using piecewise continuous software controls.

Enterprise A needs to spend its own funds $V_{1}(t)=V_{1}\left(K_{1 t}, t\right)$ at a fixed $K_{1 t}$ rate in such a way as to get the maximum at the first stage of its integral income, represented as:

$$
J_{1}(I)=\int_{t_{0}}^{T^{*}}\left[\pi_{1}^{A}(t)-V_{1}(t)\right] e^{-\mu_{1} t} d t
$$

We neglect the intermediate transformations and find, using the maximum principle of L. C. Pontryagin, the optimal time spent on switching control A, which allows maximizing (2):

$$
\begin{aligned}
& t_{11}=T^{*}-1 / \mu_{1} \ln \left\{\xi_{1} G_{1}(x) /\left(\xi_{1} G_{1}(x)-\mu_{1}\right)\right\}, \\
& 0<\mu_{1}<\xi_{1} G_{1}(x)
\end{aligned}
$$

where

$$
\begin{gathered}
G_{1}(x(t))=\partial / \partial x g_{1}(x(t))= \\
=-\left[p_{01} /\left(1+\beta_{1}\right) \varphi_{1}(x(t))^{\gamma_{1}} \varphi_{1 x}^{\prime}(x(t)) \geq 0 ;\right. \\
\gamma_{1}=\left(1+\beta_{1}\right) / \beta_{1} .
\end{gathered}
$$

To find the optimal control of A result:

$$
V_{1}^{0}(t)=\left\{\begin{array}{l}
\xi_{1} g_{1}(t)-\tau K_{1}(t)-V_{c}, \quad \text { при } t_{0} \leq t<t_{11} \\
0, \text { при } t_{11} \leq t<T^{*} .
\end{array}\right.
$$

We will solve the optimization problem for the Bank at each interval, taking into account the dynamics of switching the optimal control $\mathrm{B}$ at the first stage, $t_{01}^{*}$ and $t_{11}^{*}$ (figure 1) we will get the following relations:

$$
\begin{aligned}
& t_{11}^{*}=T^{*}-1 / \mu_{0} \ln \left\{\alpha_{B} B_{1}(x) /\left(\alpha_{B} B_{1}(x)-q_{11}\right)\right\}, \\
& t_{01}^{*}=t_{11}^{*}-1 / \mu_{0} \ln \left\{\alpha_{B}(1-\tau) B_{1}(x) /\left(\alpha_{B}(1-\tau) \times\right.\right. \\
& \left.\times B_{1}(x)-q_{11}\right)
\end{aligned}
$$

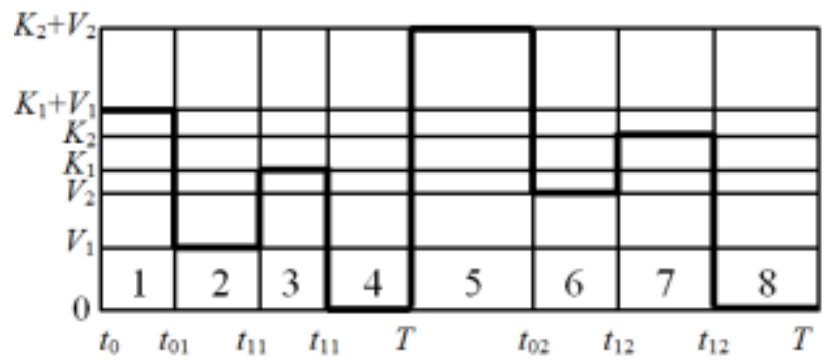

Fig. 1. Step-by-step representation of the optimization problem solution for the Bank

$$
q_{11}=\omega-\tau \alpha_{B} \geq 0, B_{1}(x)=\alpha_{B}\left(1-\xi_{1}\right) q G_{1} / \mu_{0}
$$

$q=\omega+\alpha_{B} \geq 0$, we must consider the following restrictions: $0<\mu_{0}<\alpha_{B}\left(1-\xi_{1}\right) q G_{1} / q_{11}$.

Optimal control A and B for the first stage we write:

$$
\begin{aligned}
& \left\{\begin{array}{l}
K_{1}^{0}(t)=W^{0}+(1-\xi) \alpha_{B} g_{1}(x) \text { при } t_{0} \leq t<t_{01}^{*}, t_{11} \leq t<t_{11}^{*} ; \\
K_{1}^{0}(t)=0, \text { при } t_{01}^{*} \leq t<t_{11}, \quad t_{11}^{*} \leq t<T^{*} ;
\end{array}\right. \\
& \left\{\begin{array}{l}
V_{1}^{0}(t)=\xi_{1} g_{1}(x(t))-V_{c}-\tau K_{1}^{0}(t) \quad \text { при } t_{0} \leq t<t_{11} ; \\
V_{1}^{0}(t)=0, \text { при } \quad t_{11}^{*} \leq t<T^{*} .
\end{array}\right.
\end{aligned}
$$

The integration of the system:

$$
\dot{x}(t)=\left\{\begin{array}{l}
\xi_{1} g_{1}(x(t))+(1-\tau) K_{1}^{0}(t)-V_{c}, \quad \text { при } t_{0} \leq t<t_{11}, \\
K_{1}^{0}(t), \quad \text { при } t_{11} \leq t<T^{*} ; \quad x\left(t_{0}\right)=x_{0}
\end{array}\right.
$$

allows finding the optimum for the trajectory. Let us write down a formalized representation of the maximum value of the guaranteed income criteria at the first stage, taking into account (3):

$$
\begin{aligned}
& J_{1}^{0}(I)=\xi_{1} \int_{t_{11}}^{T^{*}} g_{1}(x) e^{-\mu_{1} t} d t-\left(1-\xi_{1}\right) \alpha_{B} \tau \int_{t_{11}}^{t_{11}^{*}} g_{1}(x) e^{-\mu_{1} t} d t- \\
& -\left(1 / \mu_{1}\right)\left[\tau W^{0}\left(e^{-\mu_{1} t_{11}}-e^{-\mu_{1} t_{11}^{*}}\right)+V_{c}\left(e^{-\mu_{1} t_{11}}-e^{-\mu_{1} T^{*}}\right)\right] ;
\end{aligned}
$$




$$
\begin{aligned}
& J_{1}^{0}(I)=\tau\left(W^{0} / \mu_{0}\right)\left[\left(e^{-\mu_{0} t_{0}}+e^{-\mu_{0} t_{11}}\right)-\left(e^{-\mu_{0} t_{01}^{*}}+e^{-\mu_{0} t_{11}}\right)\right]+ \\
& +\left(1-\xi_{1}\right)\left[\tau \alpha _ { B } \left(1 / \tau \int_{t_{0}}^{T^{*}} g_{1}(x) e^{-\mu_{0} t} d t+\int_{t_{0}}^{t_{01}^{*}} g_{1}(x) e^{-\mu_{1} t} d t+\right.\right. \\
& \left.+\int_{t_{11}}^{t_{11}^{*}} g_{1}(x) e^{-\mu_{0} t} d t\right)+\omega\left(\int_{t_{01}}^{t_{11}} g_{1}(x) e^{-\mu_{0} t} d t+\int_{t_{11}}^{T^{*}} g_{1}(x) e^{-\mu_{0} t} d t\right) ;
\end{aligned}
$$

Stage II. The change for stage II is defined $x(t)=K_{2}(t)+V_{2}(t)$ with boundary conditions $x\left(T^{*}\right)=x_{02}$; $t \in\left[T^{*}, T\right], x \in X, K_{2}(t) \in K, V_{2} \in V$. The optimal control switching time for stage II is found in the same way as for the previous stage: $t_{12}$ - for enterprise $\mathrm{A}, t_{02}^{*}$ and $t_{12}^{*}$ - for Bank B (figure 1):

$$
\left.t_{12}=T-1 / \mu_{1} \ln \left\{\xi_{2}\left(1-\alpha_{c}\right) G_{2}(x) / \xi_{2}\left(1-\alpha_{c}\right) G_{2}(x)-\mu_{1}\right)\right\},
$$

where

$$
\begin{aligned}
& G_{2}(x(t))=\frac{\partial}{\partial x} g_{2}(x(t))=-\left[\frac{p_{02}}{\left(1+\beta_{2}\right) \varphi_{2}(x(t))}\right]^{\gamma_{2}} \\
& \varphi_{2}(x(t)) \geq 0, \gamma_{2}=\left(1+\beta_{2}\right) / \beta_{2},
\end{aligned}
$$

it is necessary to take into account the implementation of restrictions $0<\mu_{1}<\xi_{2}\left(1-\alpha_{c}\right) G_{2}(x)$;

$$
\begin{aligned}
& 0<\mu_{1}<\xi_{2}\left(1-\alpha_{c}\right) G_{2}(x) ; \\
& \left.t_{12}^{*}=T-1 / \mu_{0} \ln \left\{\alpha_{B} B_{2}(x) / \alpha_{B} B_{2}(x)-D_{1}\right)\right\}, \\
& \left.t_{02}^{*}=t_{12}-1 / \mu_{0} \ln \left\{\alpha_{3} B_{3}(x) / \alpha_{3} B_{3}(x)-D_{1}\right)\right\}, \\
& B_{2}(x)=\frac{D_{2} G_{2}(x)}{\mu_{2}}, \quad B_{3}(x)=\frac{q G_{2}(x)}{\mu_{0}}, \\
& D_{1}=\omega-\left(1-\alpha_{c}\right) \tau \alpha_{B}>0, \\
& D_{2}=\alpha_{B}+\left(1-\xi_{2}\right) \omega \geq 0, \\
& D_{3}=\alpha_{B}\left[1-\left(1-\alpha_{c}\right) \tau\right] \geq 0
\end{aligned}
$$

Based on (3), we find the maximum guaranteed income for stage II:

$$
\begin{aligned}
& J_{0}^{0}(I I)=\left(1-\alpha_{c}\right) \tau\left(W^{0} / \mu_{0}\right)\left[\left(e^{-\mu_{1} T^{*}}-e^{-\mu_{0} t_{12}}\right)-\left(e^{-\mu_{1} t_{02}^{*}}-e^{-\mu_{0} t_{12}^{*}}\right)\right]+ \\
& +\left[\left(1-\xi_{2}\right)(1+\tau) \alpha_{B}+\alpha_{c}\left(1-\xi_{2}\right) \tau \alpha_{B}\right]\left(\int_{T^{*}}^{*} g_{2}(x) e^{-\mu_{0} t} d t+\right. \\
& \left.+\int_{t_{12}}^{t_{12}^{*}} g_{2}(x) e^{-\mu_{0} t} d t\right)+\left[q\left(1-\xi_{2}\right)+\alpha_{c} \xi_{2}\right]\left(\int_{t_{02}^{*}}^{t_{12}} g_{2}(x) e^{-\mu_{0} t} d t+\right. \\
& \left.+\int_{t_{12}}^{T} g_{2}(x) e^{-\mu_{0} t} d t\right) ; \\
& J_{1}^{0}(I I)=\left(1-\alpha_{c}\right)\left\{\xi_{2} \int_{t_{12}}^{T} g_{2}(x) e^{-\mu_{1} t} d t-\left(1-\xi_{2}\right) \alpha_{B} \int_{t 2}^{t_{12}^{*}} g_{2}(x) e^{-\mu_{1} t} d t+\right. \\
& \left.-\tau W^{0} / \mu_{1}\left(e^{-\mu_{1} t_{12}}-e^{-\mu_{1} t_{12}^{*}}\right)\right\} ;
\end{aligned}
$$

$$
\begin{aligned}
& J_{2}^{0}(I I)=\left(1-\xi_{2}\right)\left\{\alpha_{B}\left(\int_{T^{*}}^{t_{02}^{*}} g_{2}(x) e^{-\mu_{2} t} d t+\int_{t_{12}}^{t_{12}^{*}} g_{2}(x) e^{-\mu_{2} t} d t\right)+\right. \\
& \left.+(1-q)\left(\int_{t_{02}^{*}}^{t_{12}} g_{2}(x) e^{-\mu_{2} t} d t+\int_{t_{12}^{*}}^{T} g_{2}(x) e^{-\mu_{2} t} d t\right)\right] .
\end{aligned}
$$

We will solve a boundary value problem for each obtained interval with corresponding boundary conditions (functions $\varphi_{1}(x)$ and $\varphi_{2}(x)$ explicitly set), and find the values of indicators (2) and (4). For compact sets $\mathrm{K}$ and $\mathrm{U}$ and a function $f(x)=x(t)$ of continuous bounded by $x$ and satisfying the Lipschitz condition [8], these solutions exist. Enter the appropriate symbol:

$$
I_{j}^{i}=\int_{t_{j 1}}^{t_{j i 2}} g(x) e^{-\mu_{j} t} d t \quad \text { и } \quad \lambda_{j}^{i}=-\left(1 / \mu_{j}\right)\left(e^{-\mu_{j} t_{j i 2}}-e^{-\mu_{j} t_{j i}}\right) \geq 0,
$$

$j$ - number of a group member $j=0,1,2 ; i$ is the number of interval of control $i=1, \ldots, 8 ; t_{j i 1}\left(t_{j i 2}\right)$ - the beginning (end) of the interval $i$ for party $j$, given $J_{j}^{i k}=J_{j}^{i}+J_{j}^{k}, \lambda_{j}^{i k}=\lambda_{j}^{i}+\lambda_{j}^{k}$.

Let us combine the performance indicators for the two floors and take into account (5), as well as the fact that $W^{0}=W-\alpha_{c} Q_{c}$, we will convert the maximum guaranteed income (2) and (4) for the interval $\left[t_{0}, T\right]$ to the view:

$$
\begin{aligned}
& J_{0}^{0}(T)=\tau W\left(\lambda_{0}^{13}+\lambda_{0}^{57}\right)-\alpha_{c}\left\{\tau Q_{c}\left(\lambda_{0}^{13}+\lambda_{0}^{57}\right)+\right. \\
& +Q_{c} / Q_{B}\left[\left(1-\xi_{1}\right) J_{0}^{24}+\left(1-\xi_{2}\right) J_{0}^{68}\right]- \\
& -\left(\left[\xi_{2}-\tau \alpha_{B}+\left(1-\xi_{2}\right) J_{0}^{57}+\xi_{2} J_{0}^{68}\right)+\tau W \lambda_{0}^{57}+\right. \\
& \left.+\left(\alpha_{B}+W / Q_{B}\right)\left[1-\xi_{1}\right) J_{0}^{24}+\left(1-\xi_{2}\right) J_{0}^{68}\right] ; \\
& J_{1}^{0}(T)=\alpha_{c}\left\{\tau Q_{c} \lambda_{1}^{37}+W \lambda_{1}^{7}\right)-\left[\xi_{2}-\tau \alpha_{B}\left(1-\xi_{2}\right) J_{1}^{7}-\right. \\
& \left.-\xi_{2} J_{1}^{8}\right\}-\alpha_{c}^{2} \tau Q_{c} \lambda_{1}^{7}+\left(\xi_{1} J_{1}^{34}+\xi_{2} J_{1}^{57}\right)-\tau \alpha_{B}\left(1-\xi_{1}\right) J_{1}^{3}+ \\
& \left.+\left(1-\xi_{2}\right) J_{1}^{7}\right]-\tau W \lambda_{1}^{37}-V_{c} ; \\
& J_{2}^{0}(T)=\alpha_{B}\left\{\left[\left(\left(1-\xi_{1}\right) J_{2}^{13}+\left(1-\xi_{2}\right) J_{2}^{68}\right]\right\}+\left(1+\alpha_{c}-\right.\right. \\
& \left.\left.-W / Q_{B}\right)\left[1-\xi_{1}\right) J_{2}^{24}+\left(1-\xi_{2}\right) J_{2}^{68}\right] .
\end{aligned}
$$

\section{OBJECTIVE POSSIBILITIES FOR OPTIMIZING THE MODEL FOR INVESTING INNOVATIONS IN PRODUCTION}

We will find the optimal values of $\alpha_{c}^{*}$ and $V_{c}^{*}$ that provide the appropriate level of integral profit for the group members. Let us use the appropriate symbols:

$$
\begin{aligned}
& J_{01}=a_{1} J_{0}^{57}+\xi_{2} J_{0}^{68}, \quad J_{02}=\left(1-\xi_{1}\right) J_{0}^{24}+\left(1-\xi_{2}\right) J_{0}^{68}, \\
& J_{03}=\left(1-\xi_{1}\right) J_{0}^{13}+\left(1-\xi_{2}\right) J_{0}^{57}, \\
& J_{11}=a_{1} J_{1}^{7}+\xi_{2} J_{1}^{8}, \quad J_{12}=\xi_{1} J_{1}^{34}+\xi_{2} J_{1}^{78}, \\
& J_{13}=\left(1-\xi_{1}\right) J_{1}^{3}+\left(1-\xi_{2}\right) J_{1}^{7}, \\
& J_{21}=\left(1-\xi_{1}\right) J_{2}^{13}+\left(1-\xi_{2}\right) J_{2}^{57}, \\
& J_{22}=\left(1-\xi_{1}\right) J_{2}^{24}+\left(1-\xi_{2}\right) J_{2}^{68}, \\
& a_{1}=\xi_{1}-\tau \alpha_{B}\left(1-\xi_{2}\right) \geq 0 .
\end{aligned}
$$


The maximum guaranteed returns $\mathrm{A}, \mathrm{B}$, and $\mathrm{C}$ found in (6) are recorded $\left[t_{0}, T\right]$ using the parameter functions $\alpha_{c}$ and $V_{c}$ :

$$
\begin{aligned}
& J_{0}^{0}\left(\alpha_{c}, V_{c}\right)=h_{01} \alpha_{c}+h_{01} \alpha_{c}^{2}+h_{04}, \\
& J_{1}^{0}\left(\alpha_{c}, V_{c}\right)=h_{11} \alpha_{c}+h_{12} \alpha_{c}^{2}+h_{13} V_{c}+h_{14}, \\
& J_{2}^{0}\left(\alpha_{c}, V_{c}\right)=h_{21} \alpha_{c}+h_{24} .
\end{aligned}
$$

where

$$
\begin{aligned}
& h_{01}=J_{01}-Q_{c} / Q_{B} J_{02}-\tau\left[Q_{c}\left(\lambda_{0}^{13}+\lambda_{0}^{57}\right)+W \lambda_{0}^{57}\right], \\
& h_{02}=\tau Q_{c} \lambda_{0}^{57}, \\
& h_{04}=(1+\tau) \alpha_{B} J_{03}+W / Q_{B} J_{02}+\tau W\left(\lambda_{0}^{13}+\lambda_{0}^{57}\right), \\
& h_{11}=\tau\left(Q_{c} \lambda_{1}^{37}+W \lambda_{1}^{7}\right)-J_{11}, h_{12}=-\tau Q_{c} \lambda_{1}^{7}, h_{13}=-\lambda_{1}^{34}, \\
& h_{14}=J_{12}-\tau \alpha_{B} J_{13}-\tau W \lambda_{1}^{37}, h_{21}=Q_{c} / Q_{B} J_{22}, \\
& h_{24}=\left(1-\alpha_{B}\right) J_{21}+\alpha_{2} J_{22},
\end{aligned}
$$

where $\alpha_{2}=1 / Q_{B}\left[\left(1-\alpha_{B}\right) Q_{B}-W\right]$.

Since $W \leq\left(1-\alpha_{B}\right) Q_{B}$, that $\alpha_{2} \geq 0$. Note that the function $J_{1}^{0}\left(\alpha_{c}, V_{c}\right)$ has a linear dependence on $V_{c}$ and it will reach its maximum when $V_{c}=0$.

Explicit dependency of criteria $J_{0}^{0}\left(\alpha_{c}, V_{c}\right)$ and $J_{2}^{0}\left(\alpha_{c}, V_{c}\right)$ on the parameters $V_{c}$ absent. Let $V_{c_{1}}=0$ is optimal for A. In the case of $V_{c}=V_{c_{1}}$ and implementation of requirement (3) for $W<Q_{c}$ there will be no financing of the innovation project from enterprise $A$ and it will be impossible for the innovation Fund $\mathrm{f}^{*}$ to reach the "threshold" value for a suitable period of time. Then there is a big risk in the implementation of the project. Let us assume that the managers of a corporate group will not risk their capital and will prefer a guaranteed income with minimal risk.

If we put

$$
V_{c_{0}}=\frac{1}{T^{*}}\left(1-\alpha_{c}\right) Q_{c}
$$

when $V_{c}=V_{c_{0}}$, where $V_{c_{0}}$ satisfies equation (3), the threshold $\Phi^{*}$ can be reached $V_{c}=V_{c_{1}}$ within the specified time frame. The maximum value for criterion A corresponds to the value, for $\mathrm{B}$ and $\mathrm{C}$, we consider the best $V_{c}=V_{c_{0}}$ given that their criteria do not depend on $V_{c}$. The presented justification for case A, regarding the possibility of project non-fulfillment, also holds for the situation of non-fulfillment of an innovative project, since there are no guarantees of profit for A. Given this fact, we believe that the optimal parameter $V_{c}$ value is $V_{c}=V_{c_{0}}$, guaranteeing the use of innovations in production. It is necessary to take into account, in this case, the restriction: $0 \leq V_{c 0} \leq V_{C \Pi}$, where $V_{C \Pi}$ corresponds to the minimum profit of enterprise A per unit of time, given that $g_{10}(x(t))=\xi_{1} g_{1}(x(t))$ this value corresponds $\xi_{1} g_{1}\left(x_{0}\right)$.
We assume that the value $V_{c_{0}}$ is valid if the relation

$$
0 \leq V_{c 0} \leq V_{C \Pi}, \text { where } V_{C \Pi}=\xi_{1} g_{1}\left(x_{0}\right) .
$$

The situation is different with the parameter $\alpha_{c}$, when each participant corresponds to specific values $\alpha_{c}$ (we denote them $\alpha_{c 0}, \alpha_{c 1}$ and $\alpha_{c 2}$ ) that give an extreme to their criteria. Because the

$$
W \leq Q_{c}, \text { то } 0 \leq \alpha_{c} \leq \alpha_{c \max }, \quad \text { where } \alpha_{c \max }=W / Q_{c}
$$

$W=W_{c}+W^{0}$ - reserve bank. Because $\alpha_{c}$ and $V_{c_{0}}$ are related by relation (26), it is necessary to require the fulfillment of the constraint

$$
\alpha_{c 1} \geq \alpha_{C I} ; \alpha_{C I}=\left(Q_{c}-T^{*} V_{C I}\right) / Q_{c} .
$$

The fulfillment of this condition will lead to the fulfillment of (10)

The values $\alpha_{c}$ for our model are called admissible, when $\alpha_{c} \in \Omega_{c}$, where $\Omega_{c}=\left[\alpha_{C I} ; \alpha_{\max }\right]$ is a closed bounded set.

Note that for this model, the functions $J_{0}^{0}\left(\alpha_{c}\right), J_{1}^{0}\left(\alpha_{c}\right)$ and $J_{2}^{0}\left(\alpha_{c}\right)$ are continuous differentiable with respect to $\alpha_{c}$, where $J_{0}^{0}\left(\alpha_{c}\right)$ - convex function, $J_{1}^{0}\left(\alpha_{c}\right)-$ concave, $J_{2}^{0}\left(\alpha_{c}\right)$ - linear function for any admissible $\alpha_{c}$.

The assumptions made earlier and (7) indicate continuity and differentiability with respect to $\alpha_{c}$. We differentiate the functions twice $J_{j}^{0}\left(\alpha_{c}\right), \quad j=0,1$, we get:

$$
\frac{\partial^{2}}{\partial \alpha_{c}^{2}}\left[J_{0}^{0}\left(\alpha_{c}\right)\right]=\tau Q_{c} \lambda_{0}^{57} \geq 0, \frac{\partial^{2}}{\partial \alpha_{c}^{2}}\left[J_{1}^{0}\left(\alpha_{c}\right)\right]=\tau Q_{c} \lambda_{1}^{7} \leq 0 .
$$

These inequalities are necessary and sufficient conditions for the convexity of a function $J_{0}^{0}\left(\alpha_{c}\right)$ and the concavity of a function. $J_{1}^{0}\left(\alpha_{c}\right)$.

The concavity of the function $J_{1}^{0}\left(\alpha_{c}\right)$ determines its boundedness from above and the maximum at

$$
\alpha_{c 1}=1 /\left(2 \tau Q_{c} \lambda_{1}^{7}\right)\left[\tau Q_{c} \lambda_{1}^{7}+W\right]
$$

The value $\alpha_{c_{1}}$ will be valid if for fixed $\tau, \alpha_{B}, \xi_{1}, \xi_{2}$ and $Q_{c}$ the value of the bank's $W$ resource will satisfy the following inequality:

$$
W \geq \max \left\{W_{1}, W_{2}\right\},
$$

where

$$
\begin{gathered}
W_{1} \geq\left(1 / \tau \lambda_{1}^{7}\right)\left(\tau Q_{C} \lambda_{1}^{37}-J_{11}\right) ; \\
\left.W_{2} \geq\left(1-\lambda_{1}^{3} / \lambda_{1}^{7}\right) Q_{C}+\tau \lambda_{1}^{7}-T^{*} V_{C I}\right) .
\end{gathered}
$$

Let us prove this fact. From inequality $\alpha_{c 1} \geq \alpha_{C I}$, given (13) we get: $W \geq-2 \alpha_{C I} Q_{C}+\left(1 / \tau \lambda_{1}^{7}\right)\left(J_{11}-Q_{C} \lambda_{1}^{37}\right)$, but from 
inequality $\quad \alpha_{c 1} \geq \alpha_{\max } \quad$ and

$W \geq\left(1 / \tau \lambda_{1}^{7}\right)\left(\tau Q_{C} \lambda_{1}^{37}-J_{11}\right)$

Combining these inequalities and taking into account (12), we arrive at (14). The function $J_{0}^{0}\left(\alpha_{c}\right)$ is convex; it will have a minimum at $\alpha_{c}=\alpha_{c 0}$, where

$$
\alpha_{c 0}=1 /\left(2 \tau Q_{c} \lambda_{0}^{57}\right)\left\{\left(Q_{c} / Q_{B} J_{02}+\tau\left[Q_{c}\left(\lambda_{0}^{13}+\lambda_{0}^{57}\right)+W\right]-J_{01}\right\}\right.
$$

Suppose that the value $\alpha_{c 1}$ is valid, then $J_{0}^{0}\left(\alpha_{c}\right)$ it reaches its maximum on the left or on the right border of the set $\Omega_{c}$, in accordance with the obtained value of the quantities $\alpha_{c 0}$ and $\alpha_{c 1}$. For definiteness, we assume that $\alpha_{c 0}>\alpha_{c 1}$, which is possible if the condition is met:

$$
\begin{gathered}
Q_{c} \geq 1 /\left(2 \tau Q_{c} \lambda_{0}^{57}\right)\left(\lambda_{1}^{7} J_{01}-\lambda_{0}^{57} J_{11}\right) \times \\
\times\left[J_{02}+\tau Q_{B} \lambda_{0}^{57}\left(1+\lambda_{0}^{13} / \lambda_{0}^{57}-\lambda_{1}^{37} / \lambda_{1}^{7}\right)\right]^{-1}
\end{gathered}
$$

Then the function $J_{0}^{0}\left(\alpha_{c}\right)$ reaches its maximum at $\alpha_{c}=\alpha_{C \Pi}$ (figure 2).

We believe that the use of the innovative aspect in production is advisable only when, when introduced, the effectiveness $J_{j}^{0}\left(\alpha_{c}\right), \quad j=0,1$ criterion has non-negative values for all admissible ones $\alpha_{c}$.

Let us pretend that $\Omega_{c 1}=\left[\alpha_{C I}, \alpha_{c 1}\right]$ is a closed subset of the set $\Omega_{c}, \Omega_{c 1} \subset \Omega_{c}$.

Functions $J_{j}^{0}\left(\alpha_{c}\right), \quad j=0,1$ will have non-negative values on the set $\Omega_{c 1}$, in case when $\tau, \alpha_{B}, \xi_{1}, \xi_{2}$ - fixed by parameter values $W, Q_{c}$ satisfying conditions:

$$
\begin{aligned}
& Q_{c}\left[\tau \alpha_{C I}\left(\lambda_{1}^{3}+\left(1-\alpha_{C I}\right) \lambda_{1}^{7}\right)-\left(1-\alpha_{C I}\right)+\tau \alpha_{B} J_{13}\right. \\
& \tau Q_{c} \lambda_{0}^{57}\left[\alpha_{B}(1+\tau) J_{03}+\left(\alpha_{B}+W / Q_{B}\right) J_{02}+\tau W\left(\lambda_{0}^{13}+\lambda_{0}^{57}\right)\right] \geq \\
& \geq\left\{J_{01}-Q_{c} / Q_{B} J_{02}-\tau\left[Q_{c}\left(\lambda_{0}^{13}+\lambda_{0}^{57}\right)+W \lambda_{0}^{57}\right)\right\}^{2} .
\end{aligned}
$$

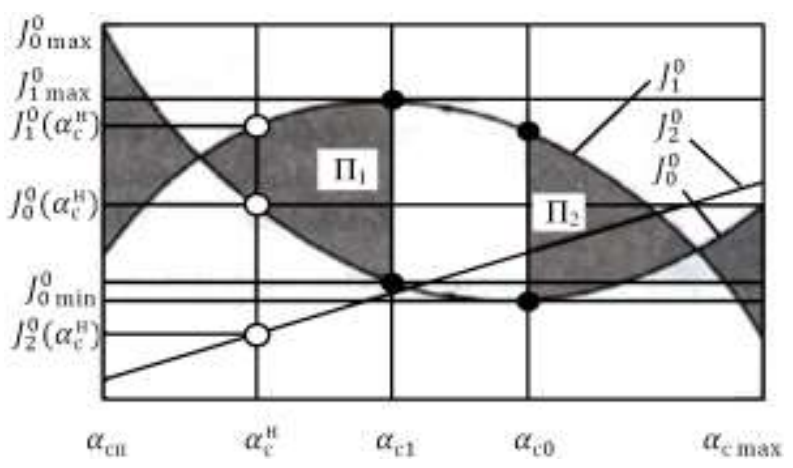

Fig. 2. The geometric interpretation of the maximum point for $J_{0}^{0}\left(\alpha_{c}\right)$

We prove this fact using (7), (8) and realizing the corresponding transformations for $\alpha_{c}=\alpha_{C \Pi}$, we find that (15) will lead to inequality $J_{1}^{0}\left(\alpha_{C \Pi}\right) \geq 0$, since it $J_{1}^{0}\left(\alpha_{C \Pi}\right)$ belongs to the ascending branch of the parabola, then, for the $\alpha_{C \Pi} \leq \alpha_{c} \leq \alpha_{c 1}$ value of the criterion $J_{1}^{0}\left(\alpha_{c_{0}}\right)$, non-negative values will also take.

Substituting (16) into (7), we obtain that when $\alpha_{c}=\alpha_{c 0}$ is fulfilled and $J_{0}^{0}\left(\alpha_{c_{0}}\right) \geq 0$, it is precisely this which will correspond to the minimum. We conclude that condition (16) guarantees the non-negativity of the function $J_{0}^{0}\left(\alpha_{c}\right)$ for any admissible one $\alpha_{c}$. Finally, the function $J_{2}^{0}\left(\alpha_{c}\right)$ increases linearly in $\alpha_{c}$, has a maximum on the right branch of $\Omega_{c}$, and $\alpha_{c}=\alpha_{\max }$ for each admissible $\alpha_{c}$, performed $J_{2}^{0}\left(\alpha_{c_{0}}\right) \geq 0$ that follows from (7).

Thus, with simultaneous fulfillment of (15) and (16) we will have: $J_{0}^{0}\left(\alpha_{c_{0}}\right) \geq 0, \quad J_{1}^{0}\left(\alpha_{c_{0}}\right) \geq 0$ and $J_{2}^{0}\left(\alpha_{c_{0}}\right) \geq 0$ for all admissible $\alpha_{c} \in \Omega_{c 1}$.

All participants are interested in such a value of the parameter $\alpha_{c} \in \Omega_{c 1}$ that delivers the maximum precisely to its criterion. In such cases, when there is a need for collective choice, they are looking for an equilibrium value that suits any participant for specific reasons $\alpha_{c}^{*}$, we will call this equilibrium value optimal.

Note that in our model, the optimum $\alpha_{c}^{*}$ (and the value of the parameter $\left.V_{c}^{*}=V_{c}\left(\alpha_{c}^{*}\right)\right)$ associated with it are determined by the choice of Bank B and Enterprise A. Enterprise C only consumes products of type $\mathrm{A}$, while it does not take part in financing the implementation of innovations, and the indicator B performance is directly dependent on the parameter $\alpha_{c}$. Therefore, the enterprise B has no possibility of influencing the choice $\alpha_{c}^{*}$ and it uses only the decisions made by the enterprises A and B. Unsatisfactory for the enterprise B will be a solution that will lead to negative values of the indicator. $J_{2}^{0}\left(\alpha_{c_{0}}\right)$. But, we have already verified that for any acceptable indicator $\alpha_{c}$ takes place $J_{2}^{0}\left(\alpha_{c_{0}}\right) \geq 0$. Therefore, each feasible solution A and B suits the enterprise B. Income matrixes A and $\mathrm{B}$ with appropriate strategies $\alpha_{c}^{0}=\alpha_{C \Pi}, \alpha_{c}^{1}=\alpha_{c 1}$, we write in the form: $\left[J_{0}^{0}\left(\alpha_{c}^{0}\right) ; J_{1}^{0}\left(\alpha_{c}^{0}\right) ;\right]\left[J_{0}^{0}\left(\alpha_{c}^{1}\right) ; J_{1}^{0}\left(\alpha_{c}^{1}\right)\right.$; ], we take into account that $J_{0}^{0}\left(\alpha_{c}^{0}\right)>J_{1}^{0}\left(\alpha_{c}^{0}\right) ; J_{0}^{0}\left(\alpha_{c}^{1}\right)>J_{1}^{0}\left(\alpha_{c}^{1}\right)$.

Pareto-optimal sets $\alpha_{C \Pi} \leq \alpha_{c} \leq \alpha_{c 1}$ and $\alpha_{c 0} \leq \alpha_{c} \leq \alpha_{c \max }$ will correspond to the region $\Pi_{1}$ and $\Pi_{2}$ (figure 2).

When $\alpha_{c_{0}}>\alpha_{c_{1}}$ many belonging to $\prod_{1}$ correspond to large values of indicators $J_{0}^{0}$ and $J_{1}^{0}$.

Between regions $\Pi_{1}$ and $S_{1}$ in the coordinate space $\left[J_{0}^{0} ; J_{1}^{0}\right]$ (figure 3) a one-to-one correspondence is established. It becomes obvious that Pareto-optimal solutions will not be unique. 


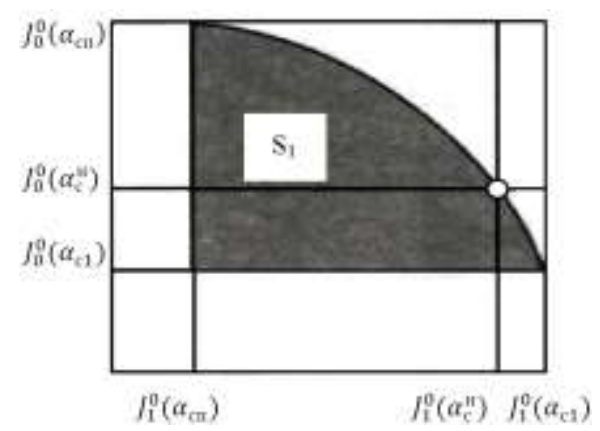

Fig. 3. Geometric interpretation of a region in a coordinate space $\left[J_{0}^{0} ; J_{1}^{0}\right]$

Note that for our model the Pareto-optimal set $S_{1}$ will be convex, closed, and bounded. This fact is confirmed by the fact that $S_{1}$ it was constructed as a result of the restrictions of functions $J_{0}^{0}\left(\alpha_{c}\right)$ and $J_{1}^{0}\left(\alpha_{c}\right)$, as well as their continuity, convexity, and concavity on a closed set of arguments $\alpha_{c}$.

Based on game theory, it is obvious that for our set there is only a single Nash equilibrium state [9-10]. If the requirement is fulfilled $J_{j}^{0}\left(\alpha_{c}^{H}\right) \geq J_{j}^{0}\left(\alpha_{c}\right), j=0,1$, the element $\alpha_{c}^{H}$ of the set $S_{1}$ determines the Nash equilibrium state. None of the players will receive additional benefits as a result of deviation from the equilibrium situation, if the player adheres to it, and this corresponds to the desire for stability.

An equilibrium strategy is defined as follows: $\alpha_{c}^{H}=\arg \left\{\max \left[J_{0}^{0}\left(\alpha_{c}\right), J_{1}^{0}\left(\alpha_{c}\right)\right]\right\}, \quad$ moreover, the product $J_{0}^{0}\left(\alpha_{c}\right)$ и $J_{1}^{0}\left(\alpha_{c}\right)$ can be represented by a polynomial of the fourth degree. The maximization condition $\frac{\partial}{\partial \alpha_{c}}\left[J_{0}^{0}\left(\alpha_{c}\right), J_{1}^{0}\left(\alpha_{c}\right)\right]=0$ will require the solution of a thirdorder equation, in which the coefficients are expressed using parameters $h_{i j}$ from (8). Found a valid root and determine the solution.

Therefore, for the analyzed model, the optimal corporate financing for the implementation of innovations, suitable for all members of the corporate group, consists in the following: at the first stage, at the time the $t_{0}$ bank, as an equity participant, invests in the introduction of innovations in the amount $W_{c}=\alpha_{c}^{*} Q_{c}$, where $\alpha_{c}^{*}=\alpha_{c}^{H}$, and enterprise $\mathrm{A}$ invests in a unit of time during the whole I stage a value $V_{c}^{*}=\frac{1}{T^{*}}\left(1-\alpha_{c}^{*}\right) Q_{c}$.

\section{INNOVATION PRODUCTIVITY}

For the presented model, we consider innovations to be economically productive if their introduction guarantees an increase in the players 'economic indicators relative to these values over the same period, but without an innovative aspect. Then, it is quite logical to consider an innovative project beyond innovation to be economically viable. We will justify the justification of the conditions for introducing innovations that require significant costs. We believe that managers of a corporate group have an option to select a project from some of their many. The main criterion for choosing a project will be considered integral indicators of the corporation's economic efficiency. The decisive value in the selection of the project is the profit function in stage II $g_{2}(x)$. We say that the use of innovations at stage I guarantees a transition $g_{1}(x)$ from a function $g_{2}(x)$ to a new function that provides higher profitability at stage II. We will present the integral performance indicators A and B (6), through the functionals $J_{1}^{0}\left(g_{1}, g_{2}\right)$ and $J_{0}^{0}\left(g_{1}, g_{2}\right)$ depending on the functions $g_{1}(x)$ and $g_{2}(x)$. If innovations were not used, then indicators $\mathrm{A}$ and $\mathrm{B}$ for the same period $\left[t_{0}, T\right]$ would have values [8]:

$$
\begin{aligned}
& \text { for A: } I_{1}^{0}(T)=\xi_{1} I_{1}^{34}-\alpha_{B} \tau\left(1-\xi_{1}\right) I_{1}^{34}-\tau W \Lambda_{1}^{3}, \\
& \text { for Б: } I_{1}^{0}(T)=\left(1-\xi_{1}\right)\left(\alpha_{B} I_{0}^{13}+q I_{0}^{24}\right)+ \\
& +\tau\left(\left(1-\xi_{1}\right) \alpha_{B} I_{0}^{13} W \Lambda_{0}^{13},\right. \\
& \text { where } \quad I_{j}^{i}=\int_{t_{i j 1}^{0}}^{t_{i j 2}^{0}} g_{1}(x) e^{-\mu_{j} t} d t \quad \text { and } \\
& \Lambda_{j}^{i}=-\left(1 / \mu_{j}\right)\left(e^{-\mu_{j} t_{i j 2}^{0}}-e^{-\mu_{j} t_{i j 1}^{0}}\right), j=0,1 .
\end{aligned}
$$

$t_{i j 1}^{0}\left(t_{i j 2}^{0}\right)$ correspond to the start (end) time of interval $i$ for participant $j$.

Suppose that $g_{0}(x)$ it is a profit function for stage II, appearing as a result of using an innovative project for which the condition is satisfied:

$$
J_{1}^{0}\left(g_{1}, g_{2}\right)=J_{j}^{0}(T), j=0,1 .
$$

We see such a project will be ineffective $\varepsilon_{1}>0, \varepsilon_{2}>0$. We assume that. If there is a project, as a result of the use of which the production function of profit in stage II (we denote it $\left.g_{2}^{*}(x)\right)$ guarantees the implementation of the condition:

$$
\Delta J_{j}=J_{j}^{0}\left(g_{1}, g_{2}^{*}\right)-J_{j}^{0}(T) \geq \xi_{j}, j=0,1 .
$$

then we will call such a project (and innovation) $\varepsilon$-effective.

We state the statement. For the constructed model, innovations will be $\varepsilon$ - effective if, as a result of their use $g_{2}^{*}(x)$, the profit function satisfies (for fixed $T^{*}, \alpha_{c}^{*}, V_{c}^{*}$ ) conditions:

$$
g_{2}^{*}(x) \geq \max \left\{g_{21}(x), g_{20}(x)\right\}
$$

where

$$
\begin{aligned}
& g_{21}(x)=g_{0}(x)+\varepsilon_{1}\left(\hat{A}_{1}\right)^{-1}, g_{20}(x)=g_{0}(x)+\varepsilon_{0}\left(\hat{A}_{0}\right)^{-1}, \\
& \hat{A}_{1}=\left(1-\alpha_{c}^{*}\right)\left\{\left[\xi_{2}-\tau \alpha_{B}\left(1-\xi_{2}\right)\right] \lambda_{1}^{7}+\xi_{2} \lambda_{1}^{8}>0,\right. \\
& \hat{A}_{0}=\left\{\alpha_{B}\left(1-\xi_{2}\right)\left\{\left[1+\tau\left(1-\alpha_{c}^{*}\right)\right]+\xi_{2} \alpha_{c}^{*}\right\} \lambda_{1}^{57}+\right. \\
& +\left[q\left(1-\xi_{2}\right)+\lambda_{0}^{68}>0, \varepsilon_{1}>0, \varepsilon_{0}>0, x \in X .\right.
\end{aligned}
$$


Check this fact. We write the profit increment A in stage II, moving to a new project, taking into account the function $g_{21}(x)$, instead of $g_{0}(x)$ as follows:

$$
\Delta J_{1}\left(g_{21}\right)=\frac{\partial}{\partial g_{2}}\left[J_{1}^{0}(I I) \Delta g_{21}\right]
$$

considering $\quad \Delta g_{21}(x)=g_{21}(x)-g_{0}(x)>0$

By analogy for B, passing from $g_{0}(x)$ to the function, $g_{21}(x)$ we obtain $\Delta J_{0}\left(g_{20}\right)=\frac{\partial}{\partial g_{2}}\left[J_{0}^{0}(I I)\right] \Delta g_{20}$, where

$$
\Delta g_{20}=g_{20}(x)-g_{0}(x)>0
$$

Derivatives $\hat{A}_{1}=\frac{\partial}{\partial g_{2}}\left[J_{1}^{0}(I I)\right], \hat{A}_{0}=\frac{\partial}{\partial g_{2}}\left[J_{0}^{0}(I I)\right]$ we find given (4):

$$
\begin{aligned}
& \hat{A}_{1}=\left(1-\alpha_{c}^{*}\right)\left(\alpha_{1} \lambda_{1}^{7}+\xi_{2} \lambda_{1}^{8}\right)>0, \\
& \text { where } \xi_{2}-\tau \alpha_{B}\left(1-\xi_{2}\right) \geq 0, \\
& \hat{A}_{0}=\left\{\alpha_{B}\left(1-\xi_{2}\right)\left[1+\tau\left(1-\alpha_{c}^{*}\right)+\xi_{2} \alpha_{B}\right] \lambda_{0}^{68}>0\right.
\end{aligned}
$$

and write the new integral revenues $A$ and $B$ as:

$$
\begin{aligned}
& J_{1}^{0}\left(g_{1}, g_{12}\right)=J_{1}^{0}\left(g_{1}, g_{0}\right)+\Delta J_{1}\left(g_{21}\right) \\
& J_{0}^{0}\left(g_{1}, g_{20}\right)=J_{0}^{0}\left(g_{1}, g_{0}\right)+\Delta J_{0}\left(g_{20}\right)
\end{aligned}
$$

As a result of substitutions (21)-(23) in (24), taking into account (18), we have conditions for each of the participants, the implementation of which will ensure the project will be $\varepsilon$ effective:

$$
\begin{gathered}
g_{21}(x)=g_{0}(x)+\varepsilon_{0}\left(\hat{A}_{1}\right)^{-1} \\
g_{20}(x)=g_{0}(x)+\varepsilon_{0}\left(\hat{A}_{1}\right)^{-1}
\end{gathered}
$$

Combining (25) and (26), we get (20). Justifying the relations (25)-(26), we assumed that the model parameters $T^{*}, \alpha_{c}^{*}, V_{c}^{*}$ remain unchanged during the transition to the evaluation of a new project in practice, it turns out that this is not always feasible. Therefore, the results obtained are appropriate for ranking projects. Only carrying out a full cycle of determining performance indicators ensures high accuracy of calculations.

\section{CONCLUSION}

The constructed model allows stating that the dynamic model of a financial and industrial corporate group with a built vertical form of integration and the existence of credit and equity relations is analyzed on a continuous time interval. As a result of the analysis, analytical dependences of the economic efficiency of such an Association are established when using innovative approaches. We have proved that the linear model of intra-corporate financing of an innovative project clearly justifies the fact that the joint use of industrial and financial capital, taking into account specific conditions, leads to an increase in the economic efficiency of such a group. The results of modeling confirm the fact that such a structure can increase the efficiency of providing financial resources to the sphere of industrial scientific research, which directly entails increasing the competitiveness of Russian products, developing a competitive economic environment while maintaining the necessary balance of interests of all participants in corporate activities. In the conditions of active formation of corporate systems, the obtained analytical results can have not only theoretical but also practical significance.

\section{References}

[1] T.E. Lebedeva, E.M. Akhmetshin, M.R. Dzagoyeva, I.S. Kobersy, S.K. Ikoev, "Corporate governance issues and control in conditions of unstable capital risk", Int. J. of Econ. and Financial Issues, vol. 6, no. 1S, pp. 25-32, 2016.

[2] C. Kwon, G. Zhang, H. Zhou, "Monetary policy, social capital, and corporate investment", J. of Econ. and Finance, vol. 44, no. 1, 2020.

[3] A.N. Ayvazli, "Scientific research on the improvement of commercial banks and application of innovations", Proc. of the Voronezh State Univer. of Engineer. Technol., vol. 81, no. 2, pp. 280-284, 2019. Retrieved from: https://doi.org/10.20914/2310-1202-2019-2-280-284

[4] M.S. Asmolova, S.V. Dedov, "The algorithm to ensure and implement the strategy of the effective management of innovation processes", Proc. of the Voronezh State Univer.of Engineer. Technol., vol. 80, no. 4, pp. 436-440, 2018. Retrieved from: https://doi.org/10.20914/2310-12022018-4-436-440

[5] M.L. Lapshina, D.D. Lapshin, A.V. Knyazev, S.V. Pisareva, V.V. Gorbunov, "Modeling the situation of non-payments on the basis of differential calculus in the enterprise integration system", Sci. J., vol. 7, no. 3. Retrieved from: http://moit.vivt.ru/ doi.org/10.26102/23106018/2019.26.3.034

[6] M. Shaverdi, S. Yaghoubi, H. Ensafian, "A multi-objective robust possibilistic model for technology portfolio optimization considering social impact and different types of financing", Appl Soft Comput J., vol. 86, 2020 .

[7] P.W. Dobson, R. Chakraborty, "Strategic incentives for complementary producers to innovate for efficiency and support sustainability", Int. J. of Product. Econ., vol. 219, pp. 431-439, 2020.

[8] O.V. Konina, "Scientific and methodological foundations of an innovative company management", Lect Notes Networks Syst., no. 91, pp. 495-502, 2020.

[9] H. Setiawan, B. Erdogan, "Key factors for successful corporate entrepreneurship: A study of indonesian contractors", Int. J. of Construct. Manag., vol. 20, no. 3, pp. 252-268, 2020.

[10] N. Natocheeva, A. Borodin, N. Rud, G. Kutsuri, M. Zholamanova, A. Namitulina, "Development of tools for realizing the potential of financial stability of enterprises", Entrepreneurship and Sustainability Issues, vol. 7, no. 2, pp. 1654-1665, 2019. 